\title{
Possíveis mecanismos trombogênicos da hiper-homocisteinemia e o seu tratamento nutricional
}

\author{
Possible thrombogenic mechanisms of \\ hyperhomocysteinemia and its nutritional treatment
}

Sofia Kimi UEHARA ${ }^{1}$

Kátia BALUZ2

Glorimar ROSA 3

\section{RE S U M O}

A hiper-homocisteinemia é um fator de risco independente para as doenças vasculares oclusivas. Os mecanismos exatos pelos quais a hiper-homocisteinemia favorece o desenvolvimento dessas doenças não são totalmente compreendidos. Sugere-se que o dano ao endotélio vascular, promovido pelas espécies de oxigênio reativas, oriundas da oxidação da homocisteína, seja a causa dos eventos tromboembólicos. Estudos indicam que a homocisteína interfere em vários mecanismos anticoagulantes mediados pelo endotélio vascular, tais como: redução da ativação da proteína $C$, inibição da trombomodulina e supressão da expressão do sulfato de heparan. Sabe-se que os níveis de homocisteína são influenciados por diversos fatores, dentre eles destaca-se a deficiência de vitaminas do complexo $B$ (especialmente folato, cobalamina e piridoxina). A suplementação de folato, isoladamente ou em combinação com a cobalamina e piridoxina, reduz os níveis de homocisteína plasmática mesmo em pessoas que não apresentam deficiência dessas vitaminas. Esta revisão tem como objetivos abordar os possíveis mecanismos pelos quais a hiper-homocisteinemia poderia levar ao desenvolvimento de eventos tromboembólicos e o tratamento nutricional da hiper-homocisteinemia.

Termos de indexação: doenças cardiovasculares, doenças vasculares, homocisteína, nutricionista.

\section{A B S T R A C T}

Hyper-homocysteinemia is an independent risk factor for occlusive vascular disease. The exact mechanisms by which hyperhomocysteinemia promotes the development of these diseases are still unclear. It has been

\footnotetext{
1 Mestranda em Nutrição Humana, Programa de Pós-Graduação em Nutrição, Instituto de Nutrição, Universidade Federal do Rio de Janeiro. Rio de Janeiro, RJ, Brasil.

2 Hospital do Câncer, Serviço de Nutrição, Instituto Nacional do Câncer. São Paulo, SP, Brasil.

3 Departamento de Nutrição e Dietética, Centro de Ciências da Saúde, Instituto de Nutrição Josué de Castro, Universidade Federal do Rio de Janeiro. Av. Brigadeiro Trompovisky, s/n, Bloco J, $2^{\circ}$ andar, Sala 24, 21949-900, Rio de Janeiro, RJ, Brasil. Correspondência para/Correspondence to: G. ROSA. E-mail: <glorimar@nutricao.ufrj.br>.
} 
suggested that damage to the vascular endothelium caused by reactive oxygen species produced during the auto-oxidation of homocysteine, may be the cause of the thrombo-embolic events. Studies have indicated that homocysteine interferes in various anticoagulant mechanisms mediated by the vascular endothelium, for example, reduction in protein C activation, inhibition of thrombomodulin and suppression of heparan sulfate expression. It is known that many factors influence the homocysteine levels including deficiency of the vitamin B complex (mainly folate, pyridoxine and cobalamin). Folate supplementation, alone or combined with pyridoxine and cobalamin, reduces plasmatic homocysteine levels even in people who are not vitamin deficient. This paper will address the possible mechanisms by which hyper-homocysteinemia could favor the development of thrombo-embolic events as well as addressing its nutritional treatment.

Indexing terms: cardiovascular diseases, vascular diseases, homocysteine, nutritionist.

\section{N T R O D U Ç Ã O}

A homocisteína é um aminoácido sulfurado que não participa da síntese protéica, sendo formada a partir do metabolismo da metionina'. Em 1969, McCully observou uma importante correlação entre os níveis aumentados de homocisteína plasmática (hiper-homocisteinemia) e a ocorrência das doenças vasculares oclusivas (infarto agudo do miocárdio, trombose e doença aterosclerótica) ${ }^{2}$. Desde então, estudos têm confirmado essa hipótese, isto é, apontam a hiper-homocisteinemia como um novo e importante fator de risco para o desenvolvimento dessas doenças ${ }^{3}$.

A concentração normal de homocisteína plasmática varia de 5 a $15 \mu \mathrm{mol} / \mathrm{L}$. A hiperhomocisteinemia pode ser assim classificada: a) moderada, entre 15 e $30 \mu \mathrm{mol} / \mathrm{L}$; b) intermediária, entre 30 e $100 \mu \mathrm{mol} / \mathrm{L}$ e c) grave, acima de $100 \mu \mathrm{mol} / \mathrm{L}^{4}$.

Segundo Okumura \& Aso ${ }^{5}$, o processo de indução das doenças vasculares oclusivas pela hiper-homocisteinemia ainda não é totalmente conhecido. Os fatores genéticos (deficiência enzimática ou mutações), patológicos (redução da função renal) e nutricionais (deficiência de folato, cobalamina e piridoxina) estão associados ao aumento dos níveis de homocisteína plasmática ${ }^{6}$.

Esta revisão visa abordar os prováveis mecanismos pelos quais a hiper-homocisteinemia poderia levar ao desenvolvimento de doenças vasculares oclusivas assim como o tratamento nutricional da hiper-homocisteinemia.

\section{Metabolismo da homocisteína}

Duas reações estão envolvidas no metabolismo da homocisteína: transulfuração e remetilação. Na reação de transulfuração, a homocisteína condensa-se irreversivelmente com a serina para formar a cistationina, sendo tal reação catalisada pela enzima cistationina $\beta$ sintase $(C \beta S)$ que é dependente de piridoxina age como um co-fator ${ }^{6}$.

Na reação de remetilação, a homocisteína é revertida a metionina ao receber o grupamento metil do 5-metiltetraidrofolato proveniente do folato dietético ${ }^{6}$. A enzima metilenotetraidrofolato redutase (MTHF redutase) é a enzima responsável pela conversão do 5,10-metilenotetraidrofolato em 5-metiltetraidrofolato. O grupo metil também pode ser doado pela betaína. A reação com o 5-metiltetraidrofolato ocorre em todos os tecidos e é dependente de cobalamina, enquanto a reação com a betaína ocorre, principalmente, no fígado e é independente de cobalamina6 (Figura 1).

\section{Associação entre a hiper-homocisteinemia e doenças cardiovasculares}

O mecanismo exato pelo qual a homocisteína exerce seu efeito aterotrombótico ainda não foi esclarecido ${ }^{8}$. Segundo Sydow \& Boger ${ }^{9}$, a disfunção endotelial pode ser a causa base para a ação pró-aterogênica associada à hiper-homocisteinemia. Entretanto, os mecanismos pelos quais a hiper-homocisteinemia promove a disfunção endotelial também não são totalmente compreendidos ${ }^{9}$. 


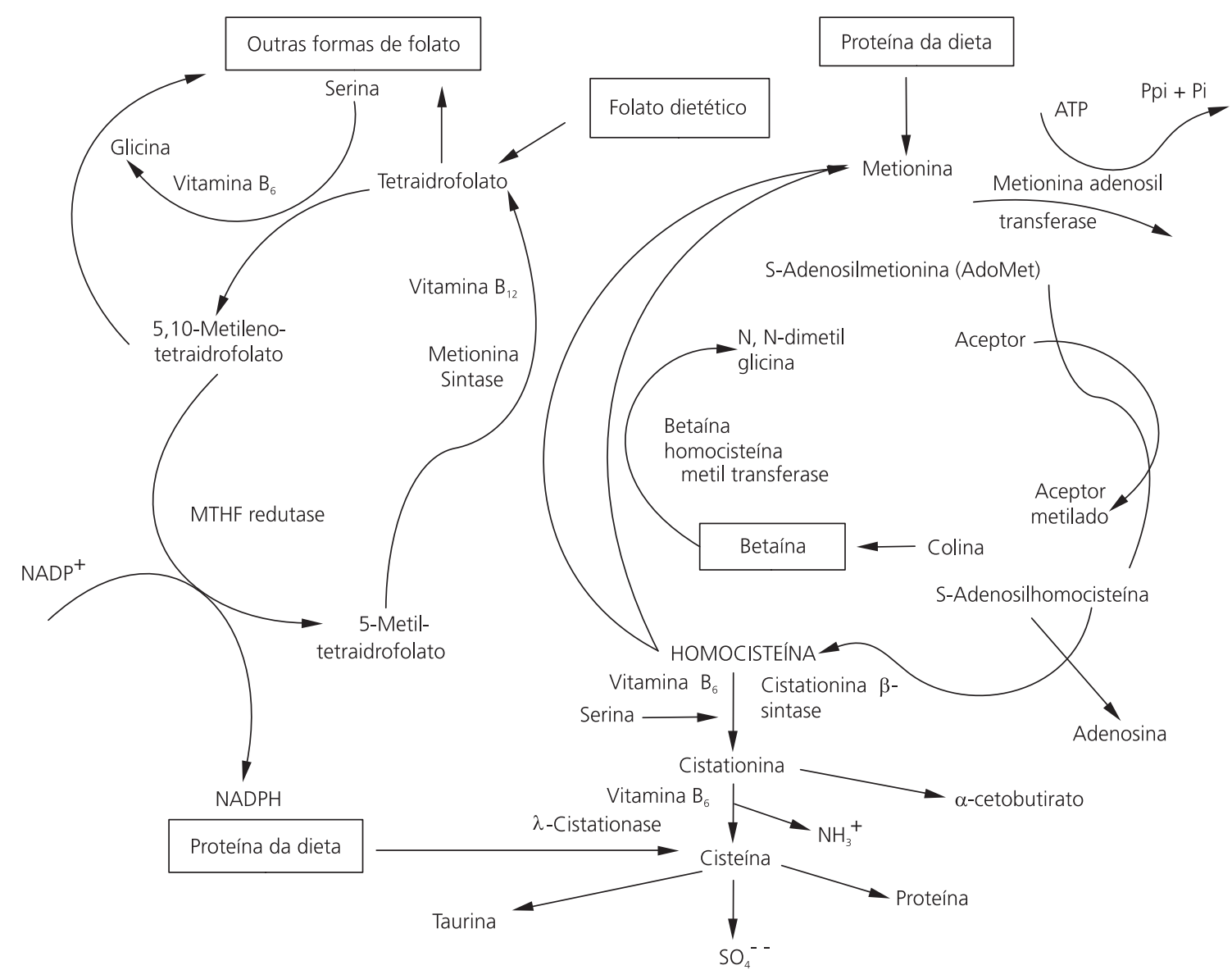

Figura 1. Metabolismo da homocisteína.

Fonte: Adaptado de Lentz ${ }^{7}$.

Segundo Weiss et al. ${ }^{10}$, a disfunção endotelial associada à hiper-homocisteinemia é manifestada pelo prejuízo da regulação do tônus vascular, do fluxo sangüíneo e perda da função antitrombótica das células endoteliais. Segundo Domagala et al. ${ }^{8}$, a produção de radicais livres durante a oxidação da homocisteína favorece a disfunção endotelial.

Quando adicionada ao plasma, a homocisteína é rapidamente auto-oxidada, originando a homocistina e homocisteína tiolactona ${ }^{11}$, bem como outras formas ativas de oxigênio consideradas citotóxicas, tais como o superóxido, peróxido de hidrogênio e radical hidroxila ${ }^{12}$. Observou-se que o radical hidroxila e o radical ânion superóxido estão envolvidos no início da peroxidação lipídica e da oxidação da lipoproteína de baixa densidade (LDL), respectivamente $^{11}$. Foi demonstrado que a homocisteína prejudica a expressão da peroxidase glutationa pelas células endoteliais, favorecendo a peroxidação lipídica ${ }^{13}$.

Segundo Domagala et al. ${ }^{8}$, a hiper-homocisteinemia estimula a proliferação de células musculares lisas e inibe o crescimento de células endoteliais, favorecendo a aterosclerose. Um dos mecanismos pelos quais a hiper-homocisteinemia favorece a proliferação de células musculares lisas é pela inativação do óxido nítrico a partir dos peróxidos de lipídios produzidos 
pela oxidação da homocisteína ${ }^{12}$. A inibição da proliferação e migração de células musculares lisas é dada pelo óxido nítrico ${ }^{12}$. A inativação do óxido nítrico deixa o endotélio mais vulnerável aos danos promovidos pela homocisteína ${ }^{12}$.

Sugere-se que o aumento moderado dos níveis de homocisteína seja o principal fator de risco para as doenças aterotrombóticas, uma vez que afeta o sistema de coagulação sangüínea ${ }^{14}$. Segundo Harpel et al. ${ }^{15}$, a hiper-homocisteinemia inibe vários mecanismos anticoagulantes que são mediados pelo endotélio vascular.

Segundo Welch et al. ${ }^{12}$, a atividade pró-coagulante do endotélio é estimulada pela hiper-homocisteinemia através dos seguintes mecanismos: aumento da atividade dos fatores $X I l$ e $V$, redução da ativação da proteína $C$, indução da expressão do fator tecidual (ativando a via extrínseca de coagulação), supressão da expressão do sulfato de heparan pelo endotélio e inibição da atividade da trombomodulina. Todos esses mecanismos facilitam a formação de trombina, aumentando a propensão de eventos trombóticos e aterogênicos ${ }^{12}$.

Lauricella et al. ${ }^{16}$ investigaram os efeitos da hiper-homocisteinemia na formação e na estrutura das fibras de fibrina e verificaram que essas fibras eram mais compactas e mais curtas, sendo mais resistentes à ação do sistema fibrinolítico. Convém destacar que a velocidade da fibrinólise sofre grande influência do tamanho e densidade das fibras. As fibras densas sofrem dissolução mais lentamente do que as finas.

Segundo Domagala et al. ${ }^{8}$, um outro possível mecanismo pelo qual a hiper-homocisteinemia pode promover a aterogênese é por meio da redução da biodisponibilidade do óxido nítrico, um potente agente antiagregante que exerce um importante papel na regulação da função plaquetária. Leoncini et al. ${ }^{17}$ observaram que a hiper-homocisteinemia leva a uma diminuição do transporte de arginina, importante para a biossíntese do óxido nítrico.
Stamler et al. ${ }^{18}$ demonstraram que as células endoteliais detoxificam a homocisteína por meio da liberação do óxido nítrico que, em presença do oxigênio, combina-se com a homocisteína, formando o S-nitroso-homocisteína. Os efeitos adversos da hiper-homocisteinemia são abrandados pela formação do S-nitroso-homocisteína que, por sua vez, não promove a formação de peróxido de hidrogênio e homocisteína tiolactona, produtos que contribuem para a toxicidade endotelial. Assim como os demais S-nitrosotióis, o S-nitroso-homocisteína é um vasodilator e inibidor plaquetário ${ }^{18}$. Entretanto, a exposição prolongada a níveis elevados de homocisteína compromete o efeito protetor mediado pelo óxido nítrico, limitando a produção do mesmo pelo endotélio ${ }^{11}$.

Segundo Harpel et al. ${ }^{15}$ e Coppola et al. ${ }^{19}$, a maioria dos estudos que investigaram os possíveis mecanismos trombogênicos da hiper-homocisteinemia empregou experimentos in vitro, nos quais foram utilizadas concentrações elevadíssimas de homocisteína; em geral, concentrações maiores do que aquelas observadas nos casos de hiper-homocisteinemia. Há poucos registros na literatura científica de estudos in vivo com humanos ${ }^{19}$.

A compreensão dos mecanismos in vivo pelos quais a hiper-homocisteinemia se relaciona com as doenças vasculares oclusivas pode fornecer novas abordagens para a prevenção e tratamento da aterotrombose ${ }^{8}$.

\section{Tratamento nutricional da hiper-homocisteinemia}

O estado nutricional de folato, cobalamina, e piridoxina parece ser o mais importante na redução dos níveis de homocisteína plasmática ${ }^{20}$. Segundo Robinson et al. ${ }^{21}$, os níveis de homocisteína plasmática aumentam à medida que os níveis plasmáticos de folato, cobalamina e piridoxina diminuem. O aumento dos níveis de homocisteína plasmática é comum em indivíduos que apresentam comprometimento do estado 
nutricional dessas vitaminas ${ }^{21}$. Segundo Ward ${ }^{22}$, a deficiência de folato, piridoxina e cobalamina é a responsável por aproximadamente dois terços de todos os casos de hiper-homocisteinemia, sendo que dessas três vitaminas, o folato parece ser o mais importante.

A deficiência das vitaminas do complexo B envolvidas no metabolismo da homocisteína é caracterizada por níveis inferiores a $372 \mathrm{nmol} / \mathrm{L}$, $125 \mu \mathrm{mol} / \mathrm{L}$ e $20 \mathrm{nmol} / \mathrm{L}$ para folato, cobalamina e piridoxina, respectivamente ${ }^{21}$.

O tratamento da hiper-homocisteinemia varia de acordo com a causa base ${ }^{11}$. Entretanto, a suplementação de folato, piridoxina e cobalamina é, em geral, eficiente na redução dos níveis de homocisteína plasmática, independente da causa da hiper-homocisteinemia ${ }^{11}$. Sugere-se que o folato, isoladamente ou em combinação com a piridoxina e a cobalamina, possa reduzir os níveis de homocisteína plasmática, mesmo em pessoas que não apresentam deficiência dessas vitaminas ${ }^{23}$. A suplementação de folato é um meio barato e eficaz na redução dos níveis de homocisteína plasmática ${ }^{23}$. A dose exata de folato, cobalamina e piridoxina necessária para o tratamento da hiper-homocisteinemia ainda não foi determinada, sendo que diferentes doses dessas vitaminas têm sido utilizadas em muitos estudos ${ }^{24}$.

Na maioria dos pacientes, pequenas doses de folato $(1000 \mu \mathrm{g}$ a $5000 \mu \mathrm{g} / \mathrm{dia})$ reduzem rapidamente os níveis de homocisteína plasmática ${ }^{11}$. Existem dados que sugerem que doses menores do que $1000 \mu \mathrm{g} / \mathrm{dia}$ de folato sejam suficientes para a redução dos níveis de homocisteína plasmática ${ }^{25}$. A administração de 200 a 15 000 $\mu \mathrm{g} / \mathrm{dia}$ de suplemento de folato pode reduzir os níveis de homocisteína plasmática sem causar toxicidade ${ }^{26}$. Considera-se que a dose diária mínima de folato que apresenta eficácia máxima na redução da homocisteína plasmática seja de aproximadamente $400 \mu \mathrm{g}^{27}$.

Em muitos casos, torna-se necessária a combinação de folato, piridoxina e cobalamina no tratamento da hiper-homocisteinemia ${ }^{24}$. A combinação de folato $(650 \mu \mathrm{g} / \mathrm{dia})$, piridoxina (10 mg/dia) e cobalamina (400 $\mathrm{gg} / \mathrm{dia})$ demonstrou ser bastante eficiente nos casos de hiperhomocisteinemia moderada ${ }^{26}$.

Schnyder et al. ${ }^{28}$ avaliaram o efeito da combinação de folato $(1000 \mu \mathrm{g})$, cobalamina $(400 \mu \mathrm{g})$ e piridoxina $(10 \mathrm{mg})$ na redução dos níveis de homocisteína plasmática em 205 pacientes submetidos à angioplastia coronariana. Verificou-se que a combinação dessas vitaminas promoveu uma redução significativa dos níveis de homocisteína plasmática, além de reduzir a ocorrência de novos eventos tromboembólicos, após a angioplastia. Embora existam outros métodos terapêuticos que possam ser utilizados para se alcançar os mesmos resultados, o baixo custo e o menor risco de complicações torna bastante freqüente a utilização dessas três vitaminas ${ }^{28}$.

Ubbink et al. ${ }^{29}$ avaliaram o efeito da suplementação de folato, piridoxina e cobalamina na redução dos níveis de homocisteína plasmática. Nesse estudo, cem indivíduos com hiper-homocisteinemia foram divididos em cinco grupos e tratados com dose diária de placebo, folato $(650 \mu \mathrm{g} / \mathrm{dia})$, cobalamina $(400 \mu \mathrm{g} / \mathrm{dia})$, piridoxina (10mg/dia) e a combinação dessas três vitaminas, durante seis semanas. Verificou-se que a suplementação de folato e de cobalamina promoveu uma redução de $41,7 \%$ e 14,8\% nos níveis de homocisteína plasmática, respectivamente. Não houve redução nos níveis de homocisteína plasmática com o uso de piridoxina. Entretanto, o grupo que recebeu a combinação das três vitaminas apresentou uma redução de 49,8\% nos níveis de homocisteína plasmática.

Brattstrom et al. ${ }^{23}$ realizaram uma metanálise de estudos que avaliaram os efeitos do folato, cobalamina e piridoxina na redução dos níveis de homocisteína. A suplementação de folato (500 a $5000 \mu \mathrm{g} / \mathrm{dia}$ ) apresentou um maior efeito, reduzindo os níveis de homocisteína em 25\%. A adição de cobalamina (20 a $1000 \mu \mathrm{g} / \mathrm{dia}$ ) promoveu um adicional de $7 \%$ na redução dos níveis de homocisteína, enquanto que a piridoxina (dose média de 16,5mg/dia) não apresentou nenhum efeito significativo. 
Nos casos em que a hiper-homocisteinemia é decorrente da deficiência de cobalamina, a mesma não responde com a terapia de folato. Desse modo, antes de iniciar o tratamento da hiper-homocisteinemia, a deficiência de cobalamina precisa ser descartada ${ }^{25}$. Recomenda-se que a ingestão máxima de folato seja de $1000 \mu \mathrm{g} / \mathrm{dia}$., uma vez que doses maiores poderiam mascarar os sinais de deficiência de cobalamina em alguns indivíduos ${ }^{26}$.

Em 1996, a Food and Drug Administration (FDA) regulamentou a fortificação de cereais matinais com folato $(140 \mu \mathrm{g} / 100 \mathrm{~g}$ de produto) com o objetivo de reduzir o risco de defeitos no tubo neural em recém-nascidos ${ }^{30}$. Em seu estudo, Jacques et al. ${ }^{30}$ verificaram que a fortificação de cereais com folato, proposta pela FDA, promoveu uma importante melhora do estado nutricional de folato. Segundo Krauss et al. ${ }^{31}$, estudos têm demonstrado que essa fortificação já tenha promovido uma redução dos níveis de homocisteína plasmática da população.

Em seu estudo, Malinow et al. ${ }^{27}$ avaliaram os efeitos do consumo de cereais matinais, fortificados com três diferentes quantidades de folato $(127 \mu \mathrm{g} / \mathrm{dia}$ - quantidade que se aproxima daquela proposta pela FDA, $499 \mu \mathrm{g} / \mathrm{dia}$ e $665 \mu \mathrm{g} / \mathrm{dia}$ ) sobre os níveis plasmáticos de folato e homocisteína. A fortificação de cereais com $127 \mu \mathrm{g} /$ dia de folato promoveu um aumento de $30,8 \%$ e redução de apenas 3,7\% nos níveis plasmáticos de folato e homocisteína, respectivamente. Os cereais matinais fortificados com $499 \mu \mathrm{g}$ e $665 \mu \mathrm{g}$ de folato promoveram um aumento de $64,8 \%$ e $105,7 \%$ nos níveis plasmáticos de folato, respectivamente, e redução de $11,0 \%$ e 14,0\% nos níveis de homocisteína plasmática, respectivamente. Segundo esses autores, os resultados do estudo sugerem que a fortificação de cereais matinais com uma quantidade de folato superior à recomendada pela FDA deve ser assegurada. Segundo Boushey et al. ${ }^{32}$, um aumento da ingestão de folato de $350 \mu \mathrm{g} /$ dia para homens e $280 \mu \mathrm{g} /$ dia para mulheres reduziria as mortes por doenças vasculares.
Riddell et al. ${ }^{33}$ verificaram que o consumo diário de cereais matinais enriquecidos com folato e o uso de suplementos dessa vitamina parecem ser os métodos mais eficientes para o aumento dos níveis de folato plasmático e a redução dos níveis de homocisteína plasmática, quando comparados com o consumo de alimentos ricos em folato. O aumento da ingestão de folato (1 $000 \mu \mathrm{g} / \mathrm{dia})$, piridoxina $(12,2 \mathrm{mg} / \mathrm{dia})$ e cobalamina $(50 \mu \mathrm{g} / \mathrm{dia})$, por meio da ingestão de alimentos fonte, não foi eficiente para a manutenção dos níveis normais de homocisteína, manutenção alcançada pelo uso de suplementos ${ }^{26}$. É improvável que a dieta isoladamente seja suficiente para aumentar os níveis circulantes de folato e reduzir os níveis de homocisteína ${ }^{34}$. A biodisponibilidade do folato dietético para a absorção intestinal é de $60 \%$ e do folato medicamentoso, $98 \%{ }^{35}$.

Os diferentes tipos de frutas e hortaliças apresentam variações no que diz respeito à biodisponibilidade do folato $^{33}$. A realização de pesquisas a respeito da biodisponibilidade do folato a partir do consumo de alimentos fonte resultará em subsídios para elaboração de estratégias governamentais para a melhora do estado nutricional de folato e redução dos níveis de homocisteína plasmática. Entretanto, é claro que recomendar o aumento do consumo de alimentos ricos em folato não é, propriamente, um meio eficiente para a redução dos níveis de homocisteína plasmática ${ }^{33}$.

Existem outros fatores que podem comprometer a biodisponibilidade do folato dietético, tais como: a estabilidade dessa vitamina nos alimentos estocados e processados industrialmente e a ação de agentes físicos, como oxidação, calor, cozimento e luz ultravioleta ${ }^{36}$.

Apesar da existência de estudos que demonstram que a ingestão de alimentos ricos em folato não seja suficiente para aumentar e diminuir os níveis de folato e homocisteína plasmáticos, respectivamente, Venn et al. ${ }^{37}$ observaram que com o aumento da ingestão de folato dietético, há uma melhora do estado nutricional 
Tabela 1. Folato, piridoxina e cobalamina em alguns alimentos.

\begin{tabular}{|c|c|c|c|c|c|}
\hline \multicolumn{2}{|l|}{ Folato $(\mu \mathrm{g} / 100 \mathrm{~g})$} & \multicolumn{2}{|l|}{ Piridoxina (mg/100g) } & \multicolumn{2}{|l|}{ Cobalamina $(\mu \mathrm{g} / 100 \mathrm{~g})$} \\
\hline Fígado de galinha cru & $=590$ & Banana & $=0,51$ & Fígado de boi cru & $=110,0$ \\
\hline Farelo de trigo & $=260$ & Abacate & $=0,42$ & Fígado de galinha cru & 56,0 \\
\hline Espinafre cozido & $=140$ & Fígado de galinha cru & $=0,40$ & Salmão cru & 5,0 \\
\hline Brócolis cozido & $=110$ & Batata cozida & $=0,18$ & Ostra cozida & 2,0 \\
\hline Farinha de aveia & $=60$ & $\mathrm{Noz}$ & $=0,17$ & Lombo de porco cru & 2,0 \\
\hline Laranja & $=37$ & Brócolis cozido & $=0,13$ & Ovo cozido & 1,7 \\
\hline Aspargo cozido & $=30$ & Farinha de aveia & $=0,12$ & Queijo parmesão & 1,5 \\
\hline Pão branco & $=27$ & Lentilha cozida & $=0,11$ & Lombo de vaca cru & 1,0 \\
\hline Banana & $=22$ & Cenoura cozida & $=0,09$ & Camarão cozido & 1,0 \\
\hline Arroz cozido & $=6$ & Alface & $=0,07$ & Leite de vaca & 0,3 \\
\hline
\end{tabular}

Fonte: Adaptado de The Composition of Foods ${ }^{38}$.

de folato e dos níveis de homocisteína plasmática a uma extensão que torna possível uma significativa redução dos riscos de doenças vasculares oclusivas.

Na Europa, a ocorrência de mortalidade por doenças vasculares oclusivas é menor nos países da região Sul, quando comparada com os países da região Norte. Segundo Ward22, o alto consumo de frutas e hortaliças pelos países da região Sul parece ser o fator que contribui para a baixa incidência de doenças vasculares oclusivas. O efeito protetor das frutas e hortaliças era atribuído à presença de antioxidantes nesses alimentos. Uma vez que as frutas e hortaliças são uma das principais fontes de folato dietético, o efeito protetor do consumo desses alimentos, na ocorrência de doenças vasculares oclusivas, poderia se dar através da redução dos níveis de homocisteína plasmática, via ingestão de folato dietético.

Segundo Malinow et al. ${ }^{26}$, a dieta da maioria da população não atinge as recomendações nutricionais de folato. Desse modo, é importante o aumento da ingestão de alimentos fontes de folato bem como de piridoxina e cobalamina. A Tabela 1 expõe o conteúdo de folato, piridoxina e cobalamina em alguns alimentos e a Tabela 2 expõe a quota diária recomendada para o folato, piridoxina e cobalamina para os indivíduos do sexo masculino e feminino.
Tabela 2. Valores de ingestão dietética de referência (IDR) para folato, piridoxina e cobalamina.

\begin{tabular}{|c|c|c|c|c|c|c|}
\hline \multirow{2}{*}{$\begin{array}{l}\text { Sexo/Faixa } \\
\text { etária (anos) }\end{array}$} & \multicolumn{2}{|c|}{$\begin{array}{l}\text { Folato } \\
(\mu \mathrm{g} / \mathrm{dia})\end{array}$} & \multicolumn{2}{|c|}{$\begin{array}{c}\text { Piridoxina } \\
\text { (mg/dia) }\end{array}$} & \multicolumn{2}{|c|}{$\begin{array}{c}\text { Cobalamina } \\
\text { ( } \mu \mathrm{g} / \mathrm{dia})\end{array}$} \\
\hline & RDA & EAR & RDA & EAR & RDA & EAR \\
\hline \multicolumn{7}{|l|}{ Homens } \\
\hline $19-30$ & 400 & 320 & 1,3 & 1,1 & 2,4 & 2,0 \\
\hline $31-50$ & 400 & 320 & 1,3 & 1,1 & 2,4 & 2,0 \\
\hline $51-70$ & 400 & 320 & 1,7 & 1,4 & 2,4 & 2,0 \\
\hline 71 e mais & 400 & 320 & 1,7 & 1,4 & 2,4 & 2,0 \\
\hline \multicolumn{7}{|l|}{ Mulheres } \\
\hline $19-30$ & 400 & 320 & 1,3 & 1,1 & 2,4 & 2,0 \\
\hline $31-50$ & 400 & 320 & 1,3 & 1,1 & 2,4 & 2,0 \\
\hline $51-70$ & 400 & 320 & 1,5 & 1,3 & 2,4 & 2,0 \\
\hline 71 e mais & 400 & 320 & 1,5 & 1,3 & 2,4 & 2,0 \\
\hline
\end{tabular}

$\mathrm{RDA}=$ quota diária recomendada; $\mathrm{EAR}=$ necessidade média estimada . Fonte: Adaptado de Institute of Medicine - Dietary Reference Intakes ${ }^{39}$.

\section{O N CLUS Ã O}

Os estudos relatam que níveis elevados de homocisteína inibem os mecanismos anticoagulantes do endotélio vascular, favorecendo a gênese de eventos tromboembólicos. É importante salientar que os estudos que investigaram essa relação foram realizados in vitro, empregando doses de homocisteína maiores daquelas observadas nos casos de hiper-homocisteinemia, o que resulta em críticas aos mesmos. Desse modo, os efeitos in vivo da hiper-homocisteinemia nos mecanismos anticoagulantes ainda não foram esclarecidos. 
Em geral, a suplementação combinada de folato, piridoxina e cobalamina promove a redução dos níveis de homocisteína plasmática. Entretanto, isoladamente, a suplementação com folato é a mais eficiente.

O estado nutricional deficiente em folato, piridoxina e cobalamina é a causa mais comum da hiper-homocisteinemia moderada. Desse modo, estimular o consumo de alimentos fontes dessas vitaminas do complexo B constitui um método simples, eficaz e econômico na prevenção da hiper-homocisteinemia e, conseqüentemente, na redução dos riscos de doenças vasculares oclusivas.

Estudos que investiguem a hiper-homocisteinemia na população brasileira, objetivando detectar sua prevalência e seus possíveis fatores determinantes, são de grande importância, permitindo ao profissional nutricionista atuar na prevenção da hiper-homocisteinemia e intervir no tratamento a fim de minimizar a ocorrência das doenças vasculares oclusivas na população brasileira.

\section{RE FER Ê N CIAS}

1. Jacques PF, Bostom AG, Wilson PWF, Rich S, Rosenberg $\mathrm{IH}$, Selhub J. Determinants of plasma total homocysteine concentration in the Framingham Offspring cohort. Am J Clin Nutr. 2001; 73(3):613-21.

2. McCully KS. Vascular pathology of homocysteinemia: implications for the pathogenesis of arteriosclerosis. Am J Pathol. 1969; 56(1):111-28.

3. Oron-Herman M, Rosenthal T, Sela B. Hyperhomocysteinemia as a component of Syndrome X. Metabolism. 2003; 52(11):1491-5.

4. Kang SS, Wong PW, Malinow MR. Hyperhomocyst(e)inemia as a risk factor for occlusive vascular disease. Ann Rev Nutr. 1992; 12:279-98.

5. Okumura K, Aso Y. High plasma homocysteine concentrations are associated with plasma concentrations of thrombomodulin in patients with type 2 diabetes and link diabetic nephropathy to macroangiopathy. Metabolism. 2003; 52(11):1517-22.
6. Saw SM, Yuan JM, Ong CN, Arakawa K, Lee HP, Coetzee GA, et al. Genetic, dietary, and other lifestyle determinants of plasma homocysteine concentrations in middle-aged and older Chinese men and women in Singapore. Am J Clin Nutr. 2001; 73(2):232-9.

7. Lentz SR. Minireview: Homocysteine and vascular dysfunction. Life Sci. 1997; 61(13):1205.

8. Domagala TB, Undas A, Libura M, Szczeklik A. Pathogenesis of vascular disease in hyperhomocysteinaemia. J Cardiovasc Risk. 1998; 5(4):239-47.

9. Sydow K, Boger RH. Homocysteine, endothelial dysfunction and cardiovascular risk: pathomechanisms and therapeutic options. Z Kardiol. 2001; 90(1):1-11.

10. Weiss N, Keller C, Hoffmann U, Loscalzo J. Endothelial dysfunction and atherothrombosis in mild hyperhomocysteinemia. Vasc Med. 2000; 7(3):227-39.

11. Welch GN, Loscalzo J. Homocysteine and atherothrombosis. N Engl J Med. 1998; 338(15): 1042-50.

12. Welch GN, Upchurch JR, Loscalzo J. Hyperhomocyst(e)inemia and atherothrombosis. Ann N Y Acad Sci. 1997; 811:48-58.

13. Upchurch GR Jr, Welch GN, Fabian AJ, Freedman JE, Johnson JL, Keaney JF Jr, et al. Homocyst(e)ine decreases bioavailable nitric oxide by a mechanism involving glutathione peroxidase. J Biol Chem. 1997; 272(27):17012-7.

14. Durand P, Prost M, Loreau N, Lussier-Cacan S, Blache D. Impaired homocysteine metabolism and atherothrombotic disease. Lab Invest. 2001; 81(5):645-72.

15. Harpel PC, Zhang X, Borth W. Homocysteine and hemostasis: pathogenetic mechanisms predisposing to thrombosis. J Nutr. 1996; 126(4):1285S-9S.

16. Lauricella AM, Quintana IL, Kordich LC. Effects of homocysteine thiol group on fibrin networks: another possible mechanism of harm. Thromb Res. 2002; 107(1-2):75-9.

17. Leoncini $G$, Pascale $R$, Signorello MG. Effects of homocysteine on L-arginine transport and nitric oxide formation in human platelets. Eur J Clin Invest. 2003; 33(8):713-9.

18. Stamler JS, Osborne JA, Jaraki O, Rabbani LE, Mullins $M$, Singel $D$, et al. Adverse vascular effects of homocysteine are modulated by endothelium-derived relaxing factor and related oxides of nitrogen. J Clin Invest. 1993; 91(1):308-18.

19. Coppola A, Davi G, De Stefano V, Mancini FP, Cerbone AM, Di Minno G. Homocysteine, 
coagulation, platelet function, and thrombosis. Semin Thromb Hemost. 2000; 26(3):243-54.

20. Kang SS. Treatment of hyperhomocyst(e)inemia: physiological basis. J Nutr. 1996; 126(4): 1273S-5S.

21. Robinson K, Arheart K, Refsum H, Brattstrom L, Boers $G$, Ueland $P$, et al. Low circulating folate and vitamin $B 6$ concentrations - risk factors for stroke, peripheral vascular disease, and coronary artery disease. European Comac Group. Circulation. 1998; 97(5):437-43.

22. Ward M. Homocysteine, folate, and cardiovascular disease. Int J Vitam Nutr Res. 2001; 71(3):173-8.

23. Brattstrom $L$, Landgren $F$, Israelsson $B$, Lindgren $A$, Hultberg B, Andersson A, et al. Lowering blood homocysteine with folic acid based supplements: meta-analysis of randomized trials. Homocysteine Lowering Trialists' Collaboration. BMJ. 1998; 316(7135):894-8.

24. Guba SC, Fink LM, Fonseca V. Hyperhomocysteinemia. An emerging and important risk factor for thromboembolic and cardiovascular disease. Am J Clin Pathol. 1996; 106(6):709-22.

25. Brattström L. Vitamins as homocysteine-lowering agents. J Nutr. 1996; 126:1276S-80.

26. Malinow MR, Bostom AG, Krauss RM. Homocyst(e)ine, diet, and cardiovascular diseases: a statement for healthcare professionals from the Nutrition Committee, American Heart Association. Circulation. 1999; 99(1):178-82.

27. Malinow MR, Duell PB, Hess DL, Anderson PH, Kruger WD, Phillipson BE, et al. Reduction of plasma homocyst(e)ine levels by breakfast cereal fortified with folic acid in patients with coronary heart disease. N Engl J Med. 1998; 338(15): 1009-15.

28. Schnyder $G$, Roffi $M$, Pin R, Flammer $Y$, Lange $H$, Eberli FR, et al. Decreased rate of coronary restenosis after lowering of plasma homocysteine levels. $\mathrm{N}$ Engl J Med. 2001; 345(22):1593-600.

29. Ubbink JB, Vermaak WJ, van der Merwe A, Becker PJ, Delport R, Potgieter HC. Vitamin requirements for the treatment of hyperhomocysteinemia in humans. J Nutr. 1994; 124(10):1927-33.
30. Jacques PF, Selhub J, Bostom AG, Wilson PW, Rosenberg $\mathrm{IH}$. The effect of folic acid fortification on plasma folate and total homocysteine concentrations. N Engl J Med. 1999; 340(19): 1449-54.

31. Krauss RM, Eckel RH, Howard B, Appel LJ, Daniels SR, Deckelbaum RJ, et al. AHA Dietary Guidelines. Revision 2000: a statement for healthcare professionals from the Nutrition Committee of the American Heart Association. Circulation. 2000; 102(18):2284-99.

32. Boushey CJ, Beresford SA, Omenn GS, Motulsky AG. A quantitative assessment of plasma homocysteine as a risk factor for vascular disease. Probable benefits of increasing folic acid intakes. JAMA. 1995; 274(13):1049-57.

33. Riddell LJ, Chisholm A, Williams S, Mann J. Dietary strategies for lowering homocysteine concentrations. Am J Clin Nutr. 2000; 71(6): 1448-54.

34. Omenn GS, Beresford SA, Motulsky AG. Preventing coronary heart disease: $B$ vitamins and homocysteine. Circulation. 1998; 97(5):421-4.

35. Baluz K, Carmo MGT, Rosa G. O papel do ácido fólico na prevenção e na terapêutica oncológica: revisão. Rev Bras Cancerol. 2002; 48(4):597-607.

36. Thurmon TF. Folic acid: miscarriages, anormalies, thromboses, cancers. J La State Med Soc. 2001; 153(2):98-103.

37. Ven BJ, Williams SM, Riddell LJ, Chisholm A, Harper MJ, Aitken W. Dietary counseling to increase natural folate intake: a randomized, placebo-controlled trial in free-living subjects to assess effects on serum folate and plasma total homocysteine. Am J Clin Nutr. 2002; 76(4):758-65

38. Paul AA, Southgate DAT. The Composition of foods. 4th ed. London; 1978.

39. Institute of Medicine. Food and Nutrition Board. Dietary Reference Intakes for thiamin, riboflavin, niacin, vitamin $B_{6}$, folate, vitamin $B_{12}$, pantothenic acid, biotin, and coline. Washington (DC): National Academy Press; 2000.

Recebido para publicação em 5 de janeiro e aceito em agosto de 2004. 